\title{
Efectos de una barrera antrópica sobre la migración río arriba del langostino anfídromo Macrobrachium tenellum (Smith 1871) (Decapoda: Palaemonidae) en la costa del Pacífico mexicano
}

\section{Effects of an anthropic barrier concerning upstream migration of the amphidromous shrimp Macrobrachium tenellum (Smith 1871) (Decapoda: Palaemonidae) in the Mexican Pacific coast}

\author{
María-Carolina Rodríguez-Uribe ${ }^{1}$, Fernando Vega-Villasante ${ }^{1 *}$, Manuel Guzmán-Arroyo ${ }^{2}$ \& Luis- \\ DANIEL EsPINOSA-ChAURAND ${ }^{1}$
}

${ }^{1}$ Laboratorio de Acuicultura Experimental. Centro de Investigaciones Costeras. Universidad de Guadalajara. Av. Universidad No. 203. Delegación Ixtapa. C.P. 48280. Puerto Vallarta, Jalisco, México.

${ }^{2}$ Instituto de Limnología. Centro Universitario de Ciencias Biológicas y Agropecuarias. Universidad de Guadalajara, México.

${ }^{3}$ Departamento de Biología. Centro Universitario de la Costa. Universidad de Guadalajara, México.

*fernandovega.villasante@gmail.com

\begin{abstract}
RESUMEN
Se evaluó el comportamiento migratorio río arriba de juveniles Macrobrachium tenellum en la compuerta de descarga libre de un estanque artificial, a través de muestreos en los meses de septiembre, octubre, noviembre y diciembre (2012) en horarios de 7,14 y $21 \mathrm{~h}(\mathrm{n}=39)$ y en un ciclo de $24 \mathrm{~h}(\mathrm{n}=10)$. El fenómeno migratorio es preponderantemente nocturno, ya que: a) la mayor abundancia de juveniles migrando es a las $0 \mathrm{~h}\left(6663 \mathrm{ind} / \mathrm{m}^{2}\right)$, mientras que la menor a las $14 \mathrm{~h}(247 \mathrm{ind} /$ $\left.\mathrm{m}^{2}\right)$; la máxima abundancia de juveniles que pasa al estanque, para continuar con la migración, es a las $2: 30 \mathrm{~h}(2700 \mathrm{ind} / \mathrm{h})$ y la mínima es de las $14 \mathrm{~h}$ a $17 \mathrm{~h}(1 \mathrm{ind} / \mathrm{h})$; de los que se desvían y mueren, debido a que pasan al área circundante, la mayor abundancia es a las $22: 30 \mathrm{~h}(4140 \mathrm{ind} / \mathrm{h})$ y la mínima a las $14 \mathrm{~h}(1 \mathrm{ind} / \mathrm{h})$ y b) existen diferencias significativas entre las abundancias de juveniles migrando en los tres horarios, con las mayores a las $21 \mathrm{~h}$ y las menores a las $14 \mathrm{~h}$ (ANOVA de una vía), además existen diferencias significativas entre los cuatro meses de observación en los tres horarios; octubre es el mes de mayores abundancias en los tres horarios (ANOVA de dos vías). Existe una correlación inversa de la abundancia de juveniles migrando con la luminosidad, se sugiere correlación con la velocidad de contracorriente, temperatura y humedad ambiental, mientras que no existe correlación con la temperatura, $\mathrm{pH}$, turbidez, salinidad y concentración de oxígeno en el agua. El ascenso por las paredes genera en los organismos estrés físico, evidenciado por una mayor concentración de glucosa $(3,23 \mathrm{mg} / \mathrm{ml})$. Los resultados obtenidos sugieren que la existencia de esta barrera tiene implicaciones negativas en el fenómeno migratorio río arriba de esta especie.
\end{abstract}

Palabras ClaVe: anfidromía, Macrobrachium, migración, presa, crustáceos.

\begin{abstract}
We evaluated the upstream migratory behavior of juvenile Macrobrachium tenellum in the discharge floodgate of an artificial pond through sampling from September through December of 2012 at 7, 14 and 21 hours ( $\mathrm{n}=39)$ and on a cycle of $24 \mathrm{~h}(\mathrm{n}=10)$. The migratory phenomenon is preponderantly at night because: a) the greatest amount of juveniles migrating is at $0 \mathrm{~h}\left(6666\right.$ organism $\left.(\mathrm{org}) / \mathrm{m}^{2}\right)$, whereas the lowest is at $14 \mathrm{~h}\left(247 \mathrm{org} / \mathrm{m}^{2}\right)$; the maximum abundance of juveniles passing to the pond to continue with the migration is at $2: 30 \mathrm{~h}(2700 \mathrm{org} / \mathrm{h})$ and the minimum is from 14 to $17 \mathrm{~h}(\mathrm{org} / \mathrm{h})$; of those which deviate and die, due to passing to the surrounding areas, the greatest abundance is at $22: 30 \mathrm{~h}(4140 \mathrm{org} / \mathrm{h})$ and the lowest at $14 \mathrm{~h}(1 \mathrm{org} / \mathrm{h})$ and $\mathrm{b})$ there are significant differences between the abundances of juveniles migrating in the three schedules, with the higher at $21 \mathrm{~h}$ and the lower at $14 \mathrm{~h}$ (one-way ANOVA). There are significant differences between the four months of observation in the three schedules; October is the month of highest abundances in the three schedules (two-way ANOVA). There exists a negative correlation of the abundance of migrating juveniles with the luminosity; correlation is suggested with the stream velocity, temperature and environmental humidity, while there is no correlation with temperature, $\mathrm{pH}$, turbidity, salinity and oxygen concentration in the water. The ascent by the walls generates physical stress on the organisms as evidenced by a higher concentration of glucose $(3,23 \mathrm{mg} / \mathrm{ml})$. These results suggest that the existence of this barrier has negative implications on the upstream migration of this species.
\end{abstract}

KEYwORDS: amphidromy, Macrobrachium, dam, migration, crustacean. 


\section{INTRODUCCIÓN}

Los crustáceos tienen una importancia mundial, no sólo por su aporte biológico y ecológico a los ecosistemas, sino también por la satisfacción de las necesidades de alimentación del hombre, ya sea como producto alimenticio directo de la pesca, como recurso fundamental en las cadenas tróficas de otros recursos bióticos y pesqueros, y como organismos de cultivo. De cualquier manera, directa o indirectamente, generan un beneficio nutrimental y/o económico a todos los usuarios en los diferentes niveles en que estos intervienen (Espinosa-Chaurand et al. 2011). Acorde con Jayachandran (2001) los crustáceos decápodos del género Macrobrachium son los más diversos de la subfamilia Palaemoninae, con más de 180 especies y subespecies en distintas partes del mundo. La distribución del género es tropical y subtropical, ocasionalmente en aguas templadas, común en aguas dulces y en ocasiones en aguas estuarinas. Las especies de langostinos de agua dulce y/o salobres que alcanzan las mayores tallas corresponden a las especies incluidas en este género (Jayachandran 2001).

De acuerdo con Bauer (2011a) en langostinos anfídromos de agua dulce, principalmente encontrados en las familias carideas: Atyidae, Xiphocarididae y Palaemonidae (mayormente en especies del género Macrobrachium), las hembras viven, se reproducen y desovan en agua dulce, pero las larvas deben ir a aguas salobres para su desarrollo. Alternativamente, las hembras ovígeras migran río abajo, con la finalidad de llevar los huevecillos lo más cerca posible de bahías costeras o estuarios. Este mismo autor menciona que tras el desarrollo larval los juveniles deben encontrar y entrar en la boca de un río o arroyo costero para migrar río arriba hasta el hábitat de agua dulce habitual, donde crecerán hasta un estadio de adultos, en ocasiones son considerables distancias. La anfidromía la define McDowall (1992) como un ciclo de vida migratorio en el cual organismos de agua dulce tienen su desarrollo larval en aguas saladas, hasta un estadio de postlarvas o juveniles, etapa en la que regresan a corrientes de agua dulce para completar sus ciclos de vida.

Los langostinos anfídromos han recibido una atención creciente en las últimas décadas, con estudios estimulados tanto por el interés fundamental en los ciclos de vida y por los impactos humanos en sus hábitats lóticos, en especial la construcción de presas (Holmquist et al. 1998) y el desvío de caudales (March et al. 2003), que impiden o detienen por completo el transporte río abajo de las larvas a aguas salobres o el posterior "retorno" migración río arriba de los juveniles (Bauer 2011a).

El langostino Macrobrachium tenellum (Smith 1871) es un organismo que se ha adaptado a ambientes diversos, se le encuentra tanto en estuarios, ríos y arroyos como en lagunas costeras, ya que tolera un amplio rango de salinidad, $\mathrm{pH}$, temperatura y oxígeno; se encuentra en grandes cantidades en la naturaleza y es una especie no agresiva (Guzmán et al. 1982). Aprovecha la época de lluvias para bajar con los caudales de los ríos y arroyos a las zonas costeras, donde las hembras desovan (Fig. 1). Su desarrollo larval se da en estas zonas salobres, hasta que alcanza la etapa de juvenil o adulto joven, el cual coincide con un aumento en la salinidad de los ambientes que habita para, posteriormente, remontarse río arriba para encontrar agua dulce y retornar a sus zonas habituales (Román-Contreras 1979, Guzmán-Arroyo 1987).

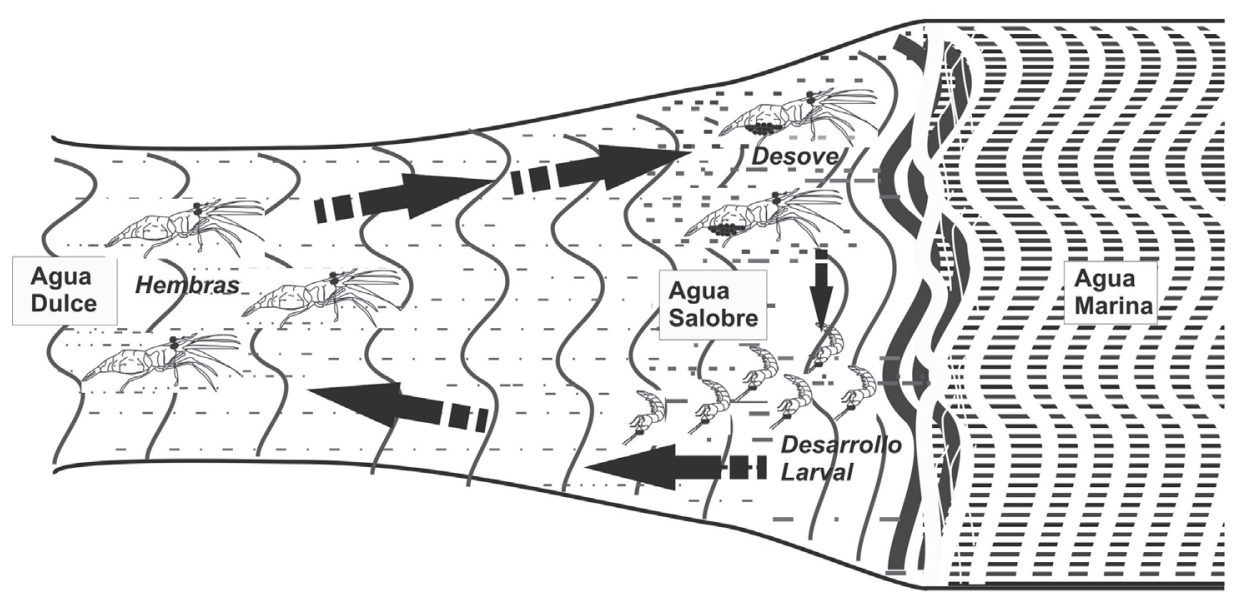

Figura 1. Migración de M. tenellum. Hembras en agua dulce (río arriba) bajan a zonas de agua salobre (estuarios) donde desovan y se da el desarrollo larval hasta el estadio de juveniles, etapa en la que inician la migración río arriba (Modificado de Boschi 1974).

Figure 1. Migration of M. tenellum. Females in freshwater (upstream) go down to areas of brackish water (estuaries) where they spawn and larval development occurs until metamorphosis to postlarva, juvenile, when they initiate the migration upstream (Modified from Boschi 1974). 
En esta migración río arriba se encuentra con impedimentos, principalmente de carácter antrópico (embalses, presas, desvío de cauces, obras civiles, contaminación del agua, pesca comercial, entre otros), los que ocasionan una disminución seria de sus poblaciones (Espinosa 1986).

En el municipio de Puerto Vallarta, Jalisco, México existen diversos ríos y arroyos que desembocan en la Bahía de Banderas, de entre estos existe un arroyo en particular, El Zarco, el cual ha sufrido diversas modificaciones antrópicas en los últimos años, una de éstas, es la creación de un estanque artificial en el Centro Universitario de la Costa, Universidad de Guadalajara (20'42'21" N/105²13'16,81" $\mathrm{W})$, transformando una sección del arroyo $(0.4 \mathrm{~km})$ de un ambiente lótico a uno léntico, cuando la compuerta del estanque artificial es cerrada para mantener el nivel de agua, lo cual es la mayor parte del año. Se han observado en años anteriores a 2012, a finales de la época de lluvias en la región (septiembre-octubre), migraciones masivas río arriba de juveniles $M$. tenellum en este arroyo, procedentes del estero El Salado (20³9'42" N/105¹4'35,36” W). Estos movimientos migratorios sufren una interrupción debido a la compuerta (reguladora del flujo de agua) de este estanque artificial. Pero, estas migraciones no han sido documentadas en este sitio, ni en otra localidad de México, por lo que surge el objetivo principal de este estudio el cual es determinar el impacto que esta barrera ejerce sobre la migración río arriba de postlarvas y juveniles del langostino anfídromo $M$. tenellum en la región.

\section{MATERIALES Y MÉTODOS}

\section{Área de ESTUdio}

Estanque artificial (20 42 '15,4" N/105 ${ }^{\circ} 13^{\prime} 16,81$ ' W) ubicado en el Centro Universitario de la Costa (CUC), Universidad de Guadalajara, con $3478.82 \mathrm{~m}^{3}$ de capacidad, aproximadamente, fondo limoso y paredes de roca con cemento. Este estanque artificial es alimentado únicamente por el arroyo El Zarco, el cual discurre $6,1 \mathrm{~km}$ desde su origen hasta el estanque. Para drenar este estanque existe una compuerta en la parte sur $\left(20^{\circ} 42^{\prime} 15,33^{\prime \prime} \mathrm{N} / 105^{\circ} 13\right.$ '19,67' $\mathrm{W})$, de $1,5 \mathrm{~m}$ por $1,3 \mathrm{~m}$ y $1,03 \mathrm{~m}$ de profundidad, una área de descarga libre de $2 \mathrm{~m}^{3}$ conectada a un tubo de concreto de $1 \mathrm{~m}$ de diámetro por $22 \mathrm{~m}$ de largo que se conecta a una sección del arroyo El Zarco, el cual fluye $252 \mathrm{~m}$ hasta unirse al arroyo Los Tamarindos que a su vez se une al estero El Salado y este desemboca en la Bahía de Banderas (Fig. 2). El sector objeto de estudio es la compuerta de descarga libre del estanque artificial, con paredes de concreto y granito, fondo de concreto y puerta de descarga de acero.

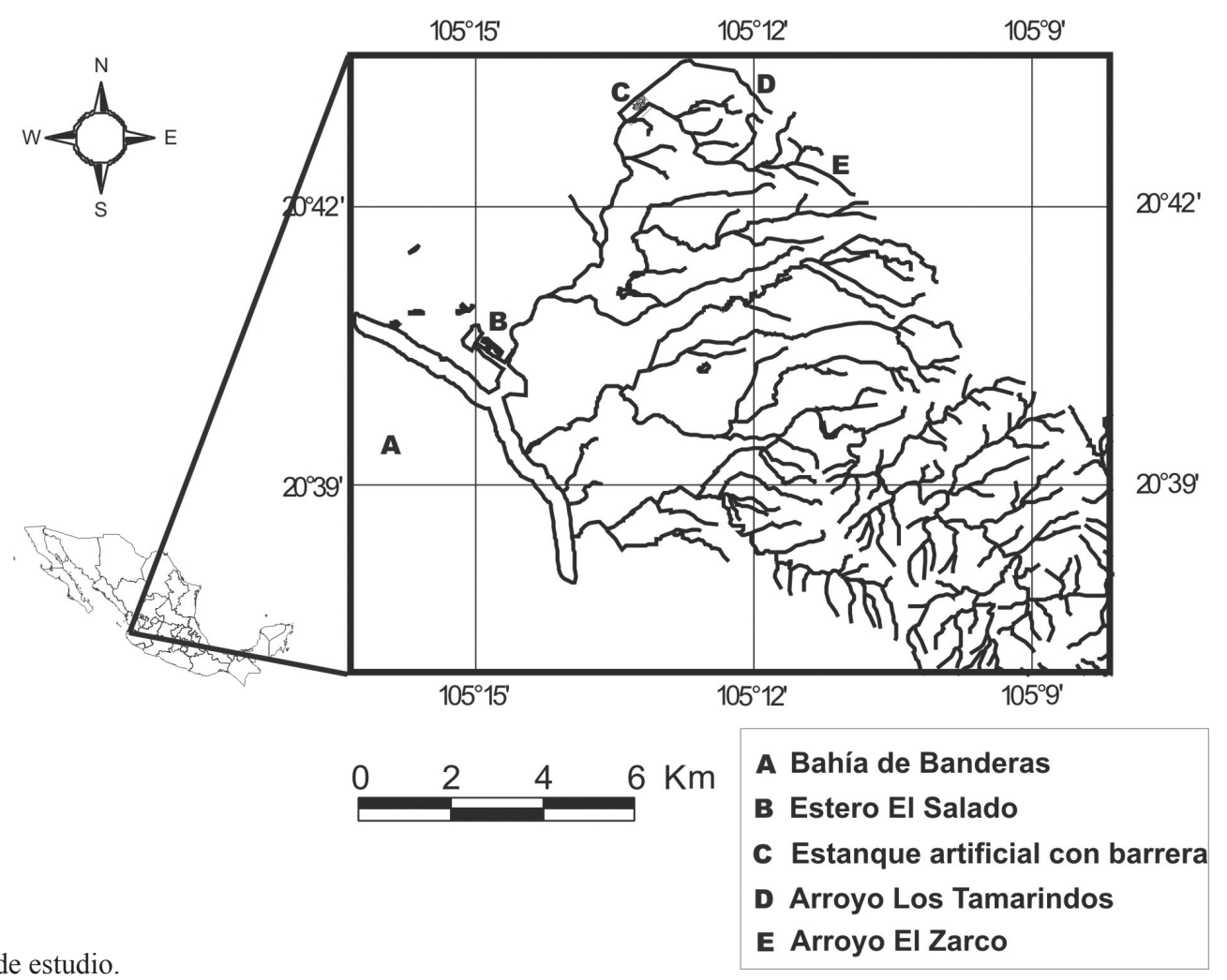

Figura 2. Área de estudio.

FIgURE 2. Study area. 


\section{Muestreos}

El período de muestreos se desarrolló durante los meses de septiembre, octubre, noviembre y diciembre del año 2012, lapso en el que se observaron postlarvas y juveniles (tallas de $17-46 \mathrm{~mm}$ ) M. tenellum migrando aguas arriba (Fig. 3a, $3 \mathrm{~b}, 3 \mathrm{c})$. El monitoreo se realizó en tres horarios $(7,14 \mathrm{y}$ $21 \mathrm{~h}$ ), con un total de 39 observaciones, además de diez observaciones obtenidas cada $3 \mathrm{~h}$ del 18 al 19 de octubre.

\section{Evaluación de la ABUNDANCIA}

En las paredes (P) laterales de la compuerta se colectaron organismos en cuadrantes de $0,2 \times 0,2 \mathrm{~m}(\mathrm{n}=42)$; en el agua (AG) se realizaron arrastres, con red de cuchara, en transectos de $0,7 \times 0,2 \mathrm{~m}(\mathrm{n}=12)$ y en el área circundante a la compuerta $(\mathrm{AC})$ se colectaron organismos en cuadrantes de $3,8 \times 1,3 \mathrm{~m}(\mathrm{n}=6)$ para obtener la abundancia relativa en cada muestreo, además de la talla de los organismos (medida desde el rostrum al telson, $\mathrm{mm}, \pm 1 \mathrm{~mm}$ ). Se contó la cantidad de organismos que lograron superar la barrera por unidad de tiempo, con ayuda de un cronómetro.

\section{Parámetros fisicoquímicos y ambientales}

Se utilizó un oxímetro (YSI $\left.{ }^{\circledR}, 550 A, 07 D 100813\right)$ para determinar la concentración de oxígeno y temperatura del agua, un potenciómetro (HANNA, HI 98129) para determinar $\mathrm{pH}$, un disco Secchi para turbidez, aforo volumétrico (tres repeticiones en cada muestra) para determinar la velocidad del flujo, luxómetro (AMPROBE, LM631A) para luminosidad. La temperatura y humedad ambiental se obtuvieron de la Estación Meteorológica Automática (EMA) del Centro de Estudios Meteorológicos de la Costa (CEMCO) ubicada en el Centro Universitario de la Costa $\left(20^{\circ} 42^{\prime} 13,86^{\prime \prime} \mathrm{N} / 105^{\circ} 13^{\prime} 20,35^{\prime \prime} \mathrm{W}\right)$.
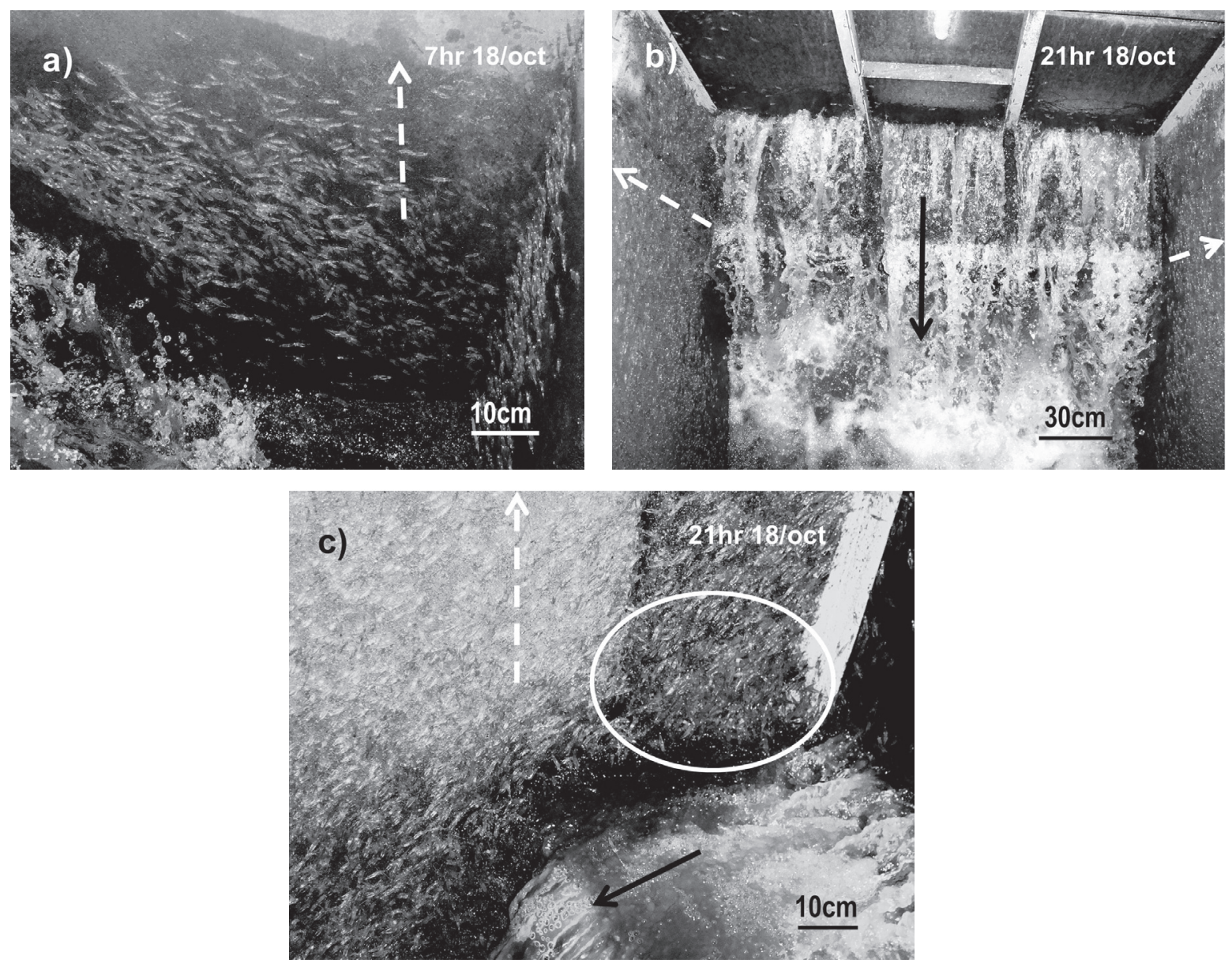

Figura 3. Migración masiva aguas arriba de juveniles M. tenellum a) Pared de la compuerta (inclinación de $90^{\circ}$ ) a las 7 h, b) Vista desde la parte superior de la compuerta $21 \mathrm{~h} \mathrm{y} \mathrm{c)} \mathrm{Conglomerado} \mathrm{de} \mathrm{juveniles} \mathrm{que} \mathrm{tratan} \mathrm{de} \mathrm{superar} \mathrm{la} \mathrm{barrera} \mathrm{dentro} \mathrm{del} \mathrm{círculo} \mathrm{blanco.} \mathrm{Las} \mathrm{flechas}$ blancas indican el movimiento migratorio de los juveniles hacia arriba en contra de la corriente (flechas negras).

Figure 3. Upstream migration of juvenile M. tenellum a) Wall of the floodgate (inclination of $90^{\circ}$ ) at $7 \mathrm{~h}$, b) View from the top of the floodgate at $21 \mathrm{~h}$ and c) Cluster of juveniles trying to overcome the barrier within the white circle. The white arrows indicate upward movement juveniles against the stream flow (black arrow). 


\section{Parámetro bioquímico}

Se colectaron 100 langostinos de cada una de las zonas de muestreo (P, AG y AC) durante la madrugada del 19 de octubre $2012(2: 30 \mathrm{~h})$, e inmediatamente después se sacrificaron por hipotermia en un congelador industrial de $15 \mathrm{ft}^{3}$ (Tor Rey) a $-18^{\circ} \mathrm{C}$ para su posterior análisis bioquímico. Se realizó la homogenización de cada muestra en frío $\left(2-4^{\circ} \mathrm{C}\right)$ con solución fisiológica de $\mathrm{NaCl}(0,9$ $\mathrm{g} / 1000 \mathrm{ml})$ a razón de $1 \mathrm{~g} / 1 \mathrm{ml}(\mathrm{p}: \mathrm{v})$. El extracto de cada muestra se centrifugó a $2000 \mathrm{G} / 4^{\circ} \mathrm{C} / 60 \mathrm{~min}$ para obtener el sobrenadante. La determinación de glucosa se realizó a través de la técnica de glucosa oxidasa (Dobrick 1958).

\section{Análisis de datos}

De las observaciones del 18 al 19 de octubre $(n=10)$ se graficó cada parámetro a lo largo del tiempo, además de obtener el Modelo de Regresión Lineal Simple (MRLS) y el coeficiente de correlación de Pearson (r), de acuerdo al método de mínimos cuadrados, tomando como variable dependiente (y) la Abundancia Relativa Total (ART) (Ind/ $\mathrm{m}^{2}$ ) y como variable independiente $(\mathrm{x})$ cada parámetro (temperatura agua, turbidez, concentración de oxígeno, $\mathrm{pH}$, luminosidad, temperatura ambiental, humedad ambiental y velocidad de contracorriente). Lo anterior con la finalidad de conocer: i) el comportamiento de cada parámetro a lo largo del tiempo, ii) cómo la ART es impactada por cada unidad que aumenta o disminuye el parámetro considerado como variable independiente $\mathrm{y}$, iii) cuál es la magnitud de la relación entre estas variables $\left(r_{x y}\right)$. De los muestreos realizados a las 7, 14 y $21 \mathrm{~h}$ de septiembre, octubre, noviembre y diciembre $(n=39)$, se graficó la Media y DE por horario y por horario de cada mes, también, se realizó un test de Análisis de Varianza (ANOVA) de una y dos vías, respectivamente, para determinar si existen diferencias entre las abundancias relativas totales entre los horarios, y entre los horarios y la estacionalidad, y se realizó el test de Tukey con una significancia del 5\%.

\section{RESULTADOS}

\section{EFEcto de La barRera SObRe LA MigraCión EN UN CICLO} DE 24HR

Se observó el fenómeno migratorio río arriba de individuos (ind) juveniles M. tenellum in situ a lo largo de $24 \mathrm{~h}$ (1819 octubre), con muestras cada $3 \mathrm{~h}$, donde se considera a la abundancia relativa de juveniles migrando y la cantidad de organismos que logran sobrepasar la barrera como las variables que explicarán el impacto que esta barrera ejerce sobre la migración río arriba en este sitio. La cantidad de organismos migrando no ocurre uniformemente a lo largo de las $24 \mathrm{~h}$, siendo los horarios nocturnos los que presentan una mayor ART, en paredes y en el cauce. La mayor ART (ind $\left./ \mathrm{m}^{2}\right)$ se observó a las $0 \mathrm{~h}\left(6663 \mathrm{ind} / \mathrm{m}^{2}\right)$, mientras que la menor a las $14 \mathrm{~h}\left(247 \mathrm{ind} / \mathrm{m}^{2}\right)$. En las paredes la mayor abundancia relativa se observó a las $2: 30 \mathrm{~h}\left(9531 \mathrm{ind} / \mathrm{m}^{2}\right)$, mientras que la mínima a las $17 \mathrm{~h}\left(96 \mathrm{ind} / \mathrm{m}^{2}\right)$, y en el cauce del canal la mayor abundancia relativa se presentó a las $22: 30 \mathrm{~h}\left(6612 \mathrm{ind} / \mathrm{m}^{2}\right)$ y la mínima a las $14 \mathrm{~h}\left(331 \mathrm{ind} / \mathrm{m}^{2}\right)$ (Fig. 4).

Las mayores cantidades de juveniles que lograron superar la barrera se observaron en los horarios nocturnos, mientras que de las $11 \mathrm{~h}$ a $17 \mathrm{~h}$ se presentaron las menores. A las 2:30 h se observó la mayor cantidad con $6420 \mathrm{ind} / \mathrm{h}$ (107 ind/ $\mathrm{min}$ ) y las menores fueron de las $11 \mathrm{~h}$ a $14 \mathrm{~h}$ con $6 \mathrm{ind} / \mathrm{h} \mathrm{y}$ de las 14 a $17 \mathrm{~h}$ con $2 \mathrm{ind} / \mathrm{h}$. Cuando los juveniles migrando in situ superaron la barrera, se observaron dos escenarios: i) lograron pasar al estanque para continuar con la migración
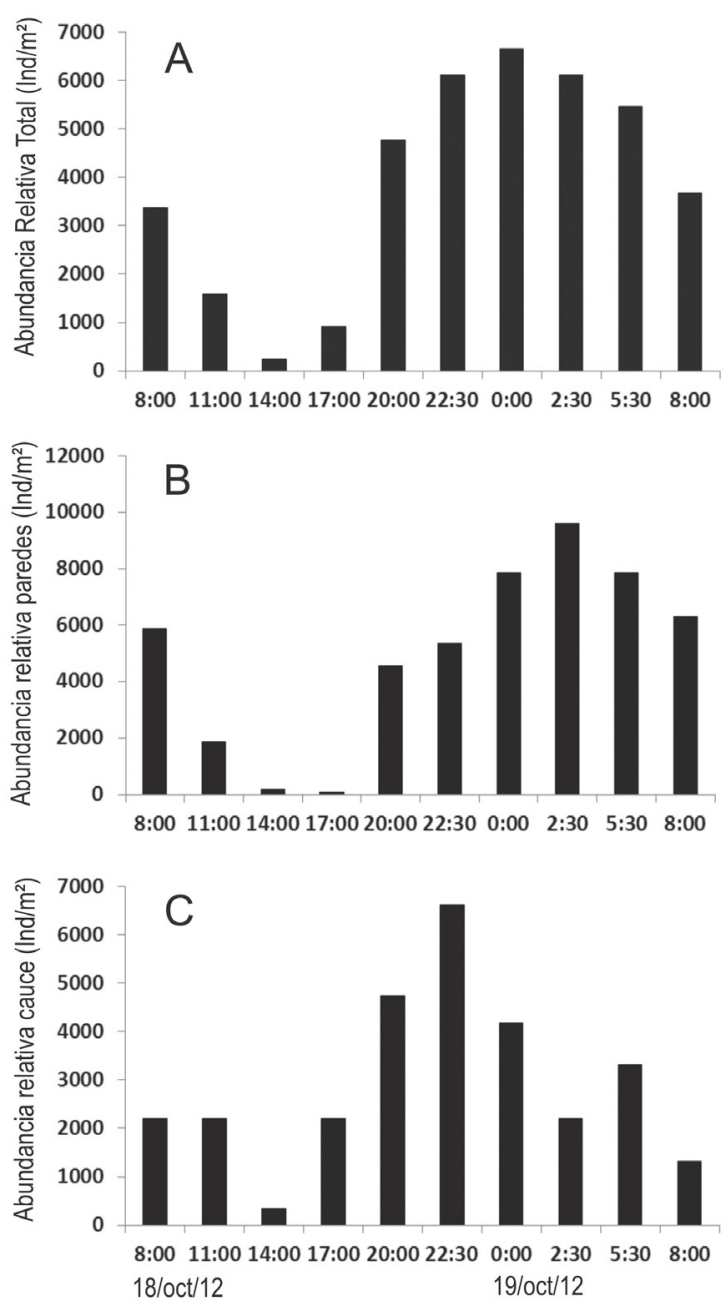

Figura 4. A. Abundancia Relativa total en el sitio de estudio, incluye individuos en paredes y en cauce por $\mathrm{m}^{2}$. B. Abundancia relativa en paredes. C. Abundancia relativa en cauce.

Figure 4. A. Total Relative Abundance in the study site individuals in the walls and the channel. B. Relative abundance per $\mathrm{m}^{2}$ on the walls. C. Relative abundance per $\mathrm{m}^{2}$ in the channel. 
o ii) se desorientaron y deambularon erráticamente por el área circundante hasta su muerte. De los que logran pasar al estanque la máxima cantidad es a las $2: 30 \mathrm{~h}$ con 2700 ind/h (45 ind/min), y la mínima es de las 14 a $17 \mathrm{~h}$ con 1 ind/h, por otra parte, la mayor cantidad de juveniles que se desvían al área circundante es de $4140 \mathrm{ind} / \mathrm{h}$ (69 ind $/ \mathrm{min})$ a las 22:30 h y la mínima a las 14 h con 1 ind/h (Fig. 5). Indicando estos datos de manera general un mayor flujo de organismos durante la noche en comparación con el flujo de organismos en el día.
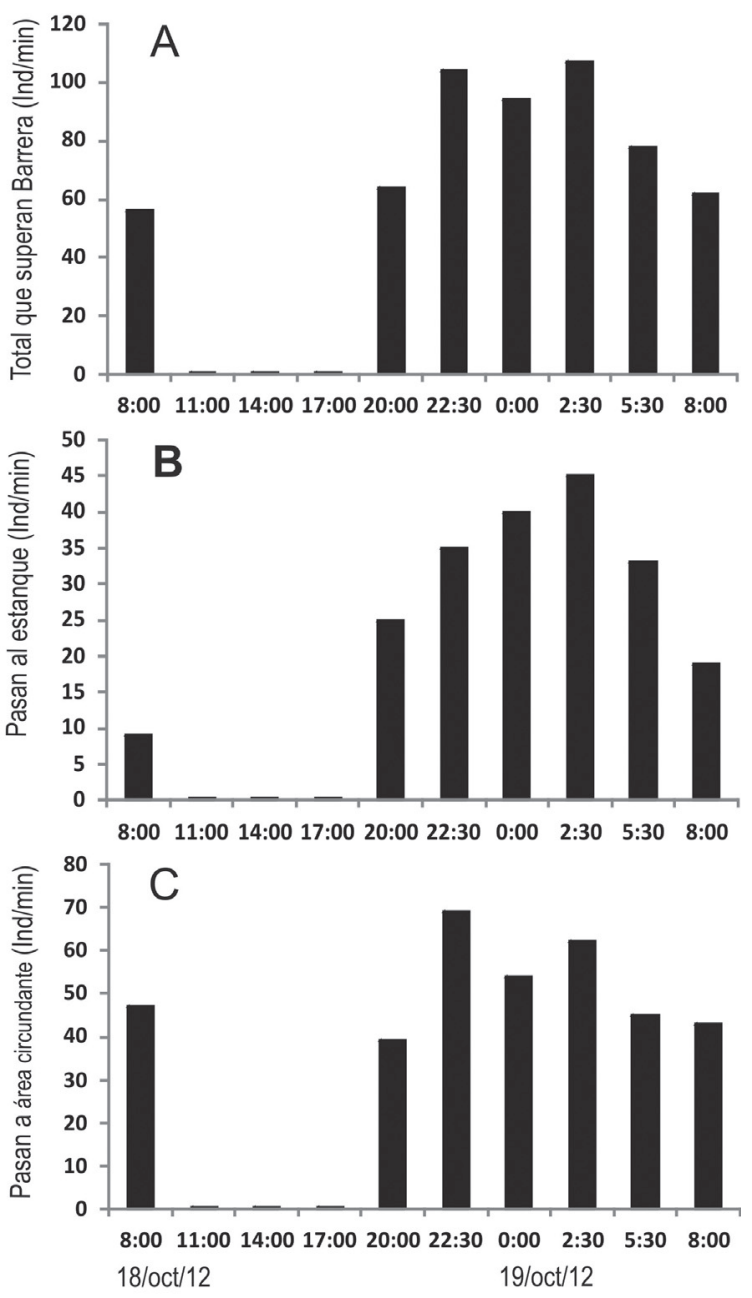

Figura 5. A. Total de juveniles que superan la barrera por minuto, de los cuales: B. Individuos que pasan al estanque, para continuar su migración río arriba y C. Individuos que pasan la barrera, pero se desvían al área circundante.

FIGURE 5. A. Total number of juveniles that overcame the barrier per minute on the night of October 19, of which: B. Those which passed into the pond to continue their upstream migration and $\mathrm{C}$. Those which passed the barrier, but deviated into the surrounding area.
Se observó en horarios nocturnos (luminosidad $=$ cero lux) una correlación inversa de la luminosidad con la ART de juveniles migrando en la barrera con un $\mathrm{r}=-0,752$, mientras que el MRLS señala que por cada lux que aumente la luminosidad, la ART disminuye en $0,84 \mathrm{ind} / \mathrm{m}^{2}$; de forma similar se observó que la temperatura ambiental tiende a disminuir cuando se acerca la noche, con un $\mathrm{r}=-0,566$ y el MRLS señala que por cada ${ }^{\circ} \mathrm{C}$ que aumente la temperatura ambiental la ART de juveniles migrando disminuye en $495,37 \mathrm{ind} / \mathrm{m}^{2}$. La velocidad de contracorriente y la humedad ambiental presentan una correlación positiva con respecto a la ART de individuos migrando en la compuerta. El MRLS de la velocidad de contracorriente arrojó que por cada $\mathrm{cm} / \mathrm{s}$ que aumente el flujo de agua la ART aumentará en 32,69 ind $/ \mathrm{m}^{2}$ con un $\mathrm{r}=0,614$; $\mathrm{y}$ el MRLS de la humedad ambiental señala que por cada unidad que aumente, la ART aumentará en $160,63 \mathrm{ind} / \mathrm{m}^{2}$, con un $\mathrm{r}=0,693$ (Fig. 6). Lo anterior sugiere una correlación de los tres últimos parámetros con la cantidad de juveniles migrando en la compuerta.

De los datos recabados en el agua (x): temperatura, turbidez, concentración de oxígeno y $\mathrm{pH}$, se observó alta dispersión en relación con la abundancia relativa total de juveniles migrando (y), así que no existe correlación $(-0,4<\mathrm{r}<0,13)$.

En los resultados bioquímicos de las muestras el menor contenido de glucosa $(2,07 \mathrm{mg} / \mathrm{ml})$ se registró en $\mathrm{AG}$, mientras que la mayor concentración se obtuvo en $\mathrm{P}(3,23$ $\mathrm{mg} / \mathrm{ml}$ ), sin embargo en AC se registró un contenido de glucosa $(2,91 \mathrm{mg} / \mathrm{ml})$ muy cercano a $\mathrm{P}$.

EFEcto de la barRera SOBRE LA MigRACión EN EL PERIOdo DE CUATRO MESES

Se observó una mayor ART en el horario de las $21 \mathrm{~h}$ (Fig. 7), mientras que la menor cantidad se observó a las 14 h; el análisis de ANOVA de una vía arrojó que existen diferencias significativas en la ART de juveniles migrando en la compuerta en los tres horarios, lo cual se comprobó con una prueba de Tukey $(\mathrm{p}=0,001)$.

Se separaron los datos por horario en cada mes, se observó que el horario de las $21 \mathrm{~h}$ presenta las máximas ART en los cuatro meses, mientras que el horario de las $14 \mathrm{~h}$ tiene las mínimas; también se observó que las máximas ART de juveniles migrando río arriba en los tres horarios $(7,14$ y 21 h) se registraron en el mes de octubre (Fig. 8); al realizar el test de ANOVA de dos vías se determinó que existen diferencias significativas entre los tres horarios y entre el mes de octubre con el resto de los meses por horario, comprobado con una prueba de Tukey $(p=0,001)$. De igual forma, como ocurre con el flujo de juveniles en el ciclo de $24 \mathrm{hr}$, estos resultados indican un mayor flujo de organismos durante la noche en comparación con el flujo de organismos en el día. 


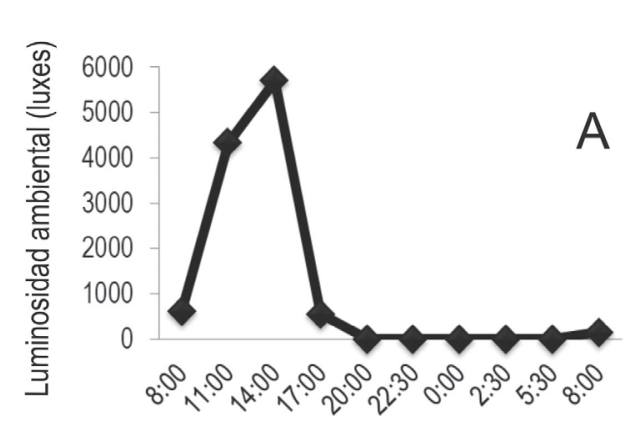

MRLS y $r$
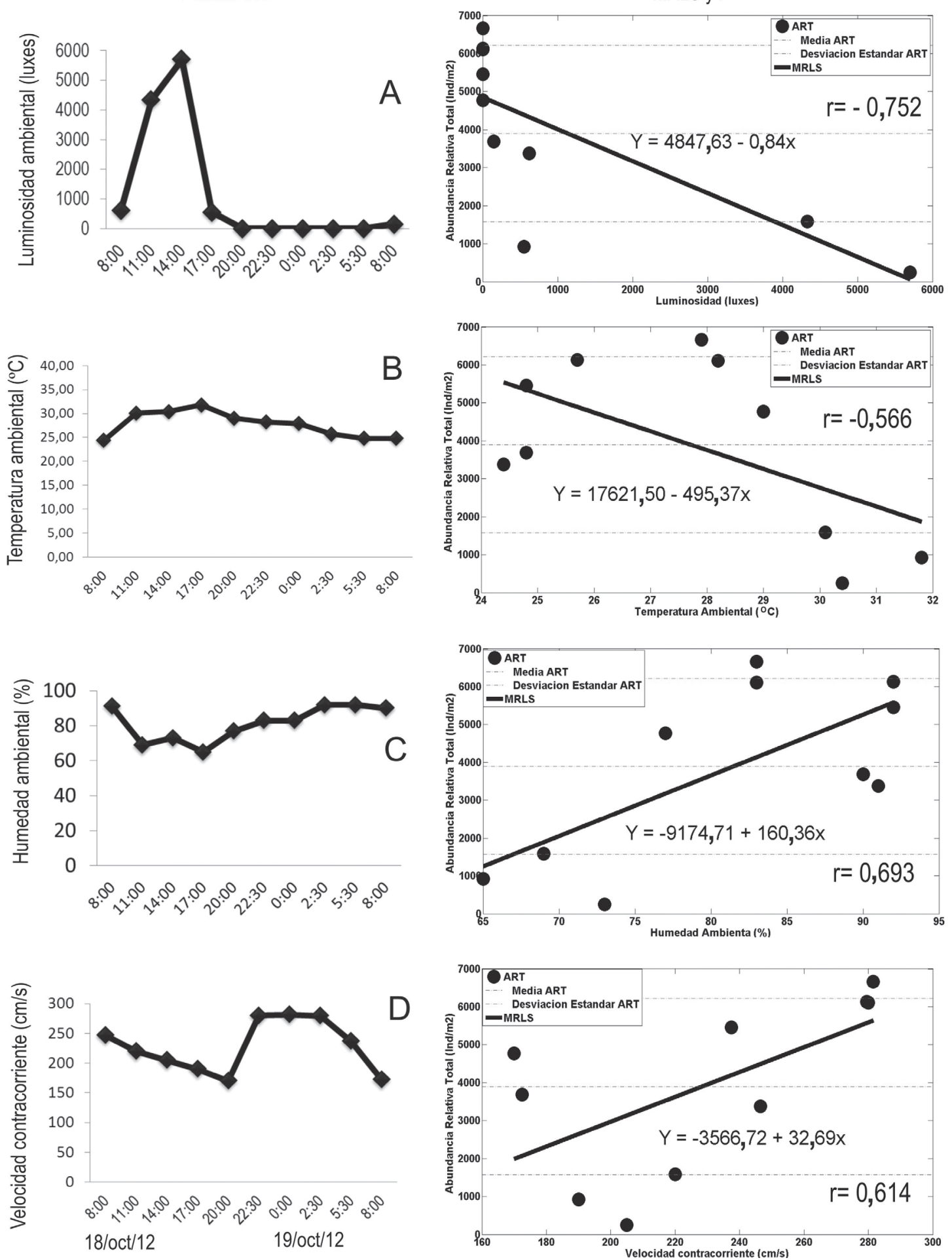

FIGURA 6. La primera columna muestra el comportamiento de cada parámetro a través del tiempo $(\mathrm{n}=10)$ y la segunda columna muestra el diagrama de dispersión de los datos, el MRLS de cada parámetro y r. A. Luminosidad ambiental. B. Temperatura ambiental. C. Humedad ambiental. D. Velocidad del flujo de agua.

FIGURE 6. The first column shows the variation of each parameter through time $(n=10)$ and the second column shows the dispersion diagram of the data, the MRLS from each parameter and $r$ between each parameter and the hour of the day. A. luminosity. B. Temperature. C. Humidity. D. Water flow rate. 


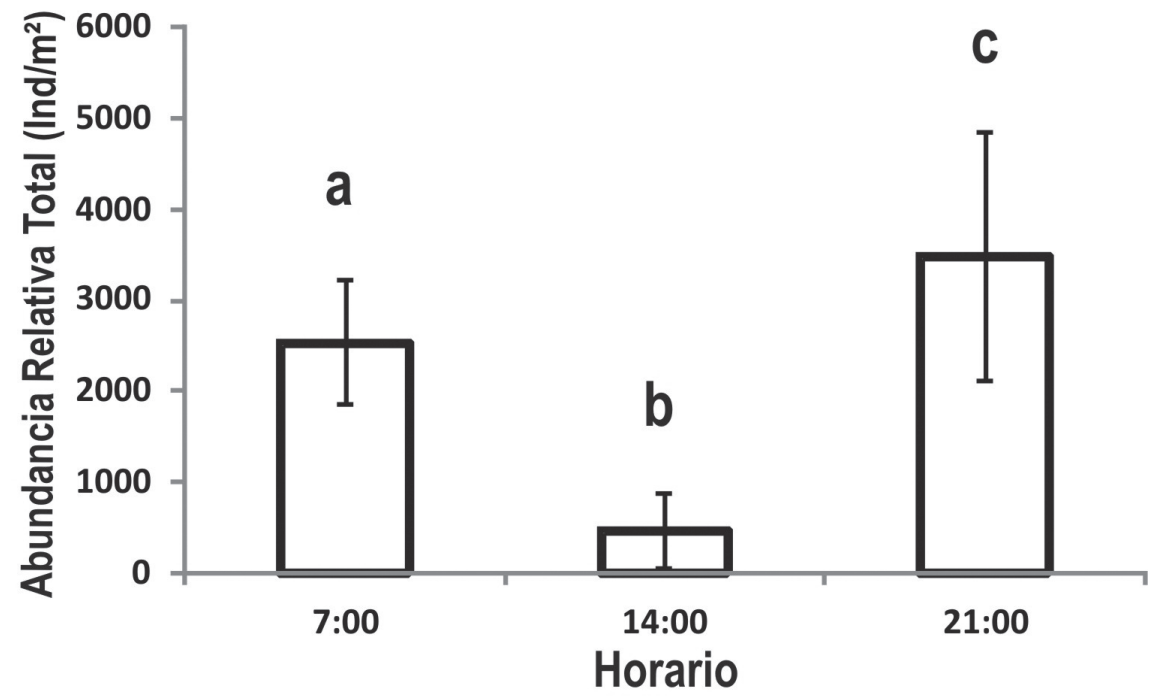

Figura 7. Abundancia Relativa Total $\left(\mathrm{Ind} / \mathrm{m}^{2}\right)$ de juveniles M. tenellum migrando en el sitio de estudio en los meses de septiembre, octubre, noviembre y diciembre $(\mathrm{n}=39)$. Las líneas verticales sobre cada barra indican la desviación estándar. Las letras sobre las columnas muestran diferencias estadísticamente significativas entre los horarios $(\mathrm{p}=0,001)$.

Figure 7. Total Relative Abundance (ind $/ \mathrm{m}^{2}$ ) of juveniles M. tenellum migrating in the study site in the months of September, October, November and December $(n=39)$. The vertical lines over each bar indicate the standard deviation. The letters over the columns show statistically significant differences between the schedules $(\mathrm{p}=0,001)$.

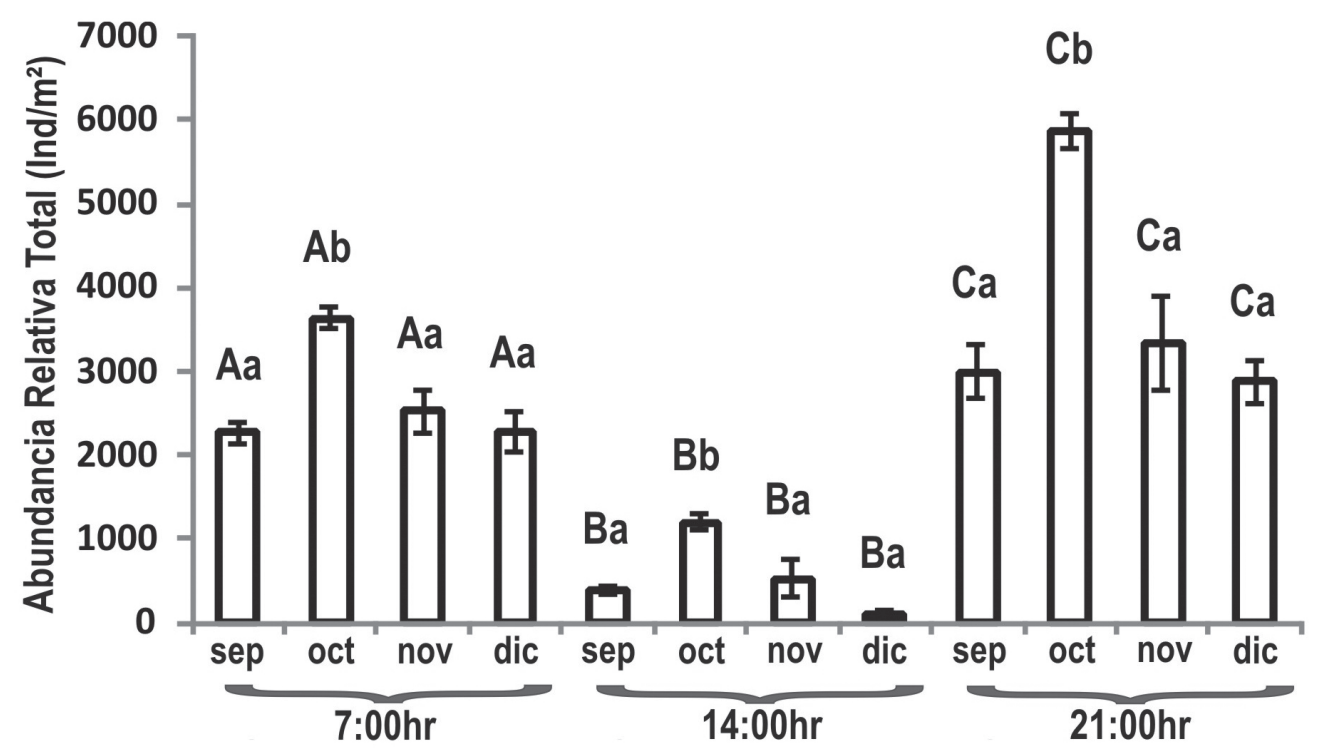

Figura 8. Abundancia Relativa Total $\left(\mathrm{Ind} / \mathrm{m}^{2}\right)$ de juveniles $M$. tenellum migrando en el sitio de estudio por horario en cada mes de observación. Las líneas verticales sobre cada barra indican la desviación estándar. Las letras mayúsculas diferentes sobre las columnas muestran diferencias estadísticamente significativas entre los horarios $(\mathrm{p}=0,001)$. Las letras minúsculas diferentes sobre las columnas muestran diferencias estadísticamente significativas entre los meses por cada horario $(\mathrm{p}=0,001)$.

Figure 8. Total Relative Abundance (Ind/ $\mathrm{m}^{2}$ ) of juveniles $M$. tenellum migrating in the study site by schedule in each month of observation. The vertical lines over each bar indicate the standard deviation. The different capital letters on the columns show statistically significant differences between the schedules $(p=0,001)$. The different lowercase letters over the columns show statistically significant differences between the months for each schedule $(\mathrm{p}=0,001)$. 


\section{DISCUSIÓN}

Se observó que las mayores abundancias de postlarvas y juveniles $M$. tenellum migrando río arriba en el área de interés se presentan en las horas nocturnas, hecho observado igualmente en diversas especies de crustáceos decápodos anfídromos: Macrobrachium malcomsonii (Ibrahim 1962), Caridina japónica (Hamano \& Hayasahi 1992), Atya innocuous, A. lanipes, A. scabra, Micratya poeyi, Macrobrachium carcinus, M. crenulatum, M. faustinum, M. heterochirus y Xiphocaris elongata (Benstead et al. 1999), Macrobrachium ohione (Bauer \& Delahoussaye 2008) y Atya innocuous, A. lanipes, A. poeyi, A. scabra, Macrobrachium acanthurus, M. carcinus, M. crenulatum, M. faustinum, M. heterochirus y Xiphocaris elongata (Kikkert et al. 2009). Existe una correlación negativa de la abundancia de juveniles $M$. tenellum, migrando río arriba en el área, con la intensidad luminosa $(\mathrm{r}=-0,752)$, resultado que concuerda con lo observado por Kikkert et al. (2009) en especies de tres familias de decápodos: Atyidae, Palaemonidae y Xiphocarididae; ya que consideran que la reducción de la intensidad luminosa es el factor decisivo de que el fenómeno migratorio sea preponderantemente en la noche, evitando así la depredación por caza visual de peces y aves. De acuerdo a los análisis de regresión lineal que se realizaron en este estudio se infiere que podría existir correlación entre la abundancia de juveniles $M$. tenellum migrando río arriba con la velocidad de la contracorriente $(\mathrm{r}=0,614)$, con la humedad ambiental $(\mathrm{r}=0,693)$ y con la temperatura ambiental $(\mathrm{r}=-0,566)$; mientras que no existe correlación con la temperatura, $\mathrm{pH}$, turbidez y concentración de oxígeno en el agua. Por otra parte, Kikkert et al. (2009) analizaron la influencia de la nubosidad y la luz de la luna sobre las migraciones de juveniles de tres familias de decápodos y no siempre encontraron los efectos positivos o negativos esperados.

Durante los muestreos de septiembre, octubre, noviembre y diciembre en la compuerta se observaron juveniles $M$. tenellum con tallas de $17-46 \mathrm{~mm}$, pero en los dos últimos se observaron tallas de hasta $59 \mathrm{~mm}$, una consecuencia del rezago de individuos en sobrepasar la barrera. Durante el día se observó que los juveniles se ubicaban a los costados del tubo de concreto (vía de entrada de los organismos a la compuerta), con el cuerpo total o parcialmente bajo el agua. Las condiciones ambientales dentro del tubo no fueron registradas (se sugiere además de la oscuridad una mayor humedad ambiental), la velocidad de la corriente se estableció como menor en los costados del mismo que en el centro. De acuerdo con Hartmann (1958), Bauer \& Delahoussaye (2008), Kikkert et al. (2009) y Bauer (2011b) durante el día los juveniles de varias especies de anfídromos pueden descansar, alimentarse y mudar en un hábitat protegido a lo largo de la orilla del río o arroyo. Esto último sugerido por el aumento en la talla (crecimiento) al aumentar la distancia río arriba desde el mar. Las observaciones realizadas en el presente estudio aparentemente concuerdan con lo antes citado.

Con relación al comportamiento de los juveniles $M$. tenellum frente a la barrera, se observó: a) Los juveniles escalan las paredes de la compuerta $\left(90^{\circ}\right)$ sujetándose con el segundo par de pereiópodos y utilizando como apoyo los restantes. b) En los momentos de mayor presencia de organismos ascendiendo, la posibilidad de salvar la barrera se vio afectada por la gran acumulación de individuos en un mismo lugar lo que ocasionaba la formación de capas de organismos (unos arriba de otros), hasta que el peso de los mismos los hacía caer de nuevo al agua. c) El regreso al agua de los juveniles que cayeron intentando subir las paredes no es factor limitante para que los mismos no intenten un nuevo ascenso. En el $100 \%$ de los casos lo reanudaron. d) Los organismos con tallas menores a $30 \mathrm{~mm}$ tienen una mayor capacidad para ascender y salvar la barrera. e) De los juveniles que logran superar la barrera el $42 \%$ pasa directamente al estanque artificial para continuar su migración río arriba, mientras que el resto $(58 \%)$ se desvía al área circundante, donde mueren por deshidratación o por depredación de las aves del lugar.

El fenómeno migratorio en el sitio de estudio inició a finales (septiembre) del período de lluvias en la región llega a su clímax en el mes de octubre, llegando a $9531 \mathrm{ind} / \mathrm{m}^{2}$, e inicia el descenso en noviembre y diciembre, éstos salían de los costados del tubo de concreto y de las orillas del cauce. De acuerdo a Bauer (2011b) una hipótesis obvia para explicar el movimiento migratorio de juveniles a las orillas de los ríos, arroyos o corrientes, es que la velocidad de la corriente es menor en esta zona y les requiere menor cantidad de energía para moverse en contra de ésta. Este fenómeno concuerda con los resultados de concentración de glucosa obtenidos en este estudio: los langostinos muestreados en el curso de agua (AG) registraron una menor concentración de glucosa que aquellos enfrentados al estrés físico de intentar superar la barrera. Jaime Ceballos et al. (2008) mencionan que cuando los camarones están estresados, la glucosa de la sangre se incrementa para ser usada como una fuente rápida de energía (metabolismo de huida). Bajo las circunstancias específicas de este trabajo, el esfuerzo físico mantenido por los langostinos durante periodos prolongados, sugiere que los niveles de glucosa sanguínea se elevaron sobre sus niveles basales como respuesta metabólica a la necesidad de energía disponible. La imposibilidad, en la mayoría de los casos, de lograr rebasar la barrera en el primer intento sometió a los organismos a condiciones de agotamiento y por consecuencia estrés. El agotamiento físico extremo al que se ven sometidos estos juveniles podría implicar un desgaste en sus capacidades de respuesta a la depredación 
o inclusive al hecho de poder o no completar su ciclo migratorio, aunque ya hayan logrado superar la barrera.

Se sugiere que la migración río arriba de estos juveniles está siendo afectada negativamente por la existencia de la compuerta de descarga libre del estanque artificial, tal conclusión se basa en las siguientes evidencias: a) El 58\% de los organismos que logran superar la barrera muere por deshidratación o por depredación de aves; b) Los juveniles que se encuentran dentro de la compuerta están expuestos y son depredados por: aves, reptiles y peces; c) La actividad de escalar las paredes de la barrera es causa de estrés físico en los organismos, evidenciado por el aumento de la concentración de glucosa; d) Visualmente se aprecian modificaciones en el exoesqueleto de los organismos que logran superar la barrera (pérdida de brillantez y textura), y además en muchos de ellos el color del músculo adquiere un color blanquecino. Este cambio en la coloración se produce por la necrosis de las fibras musculares (Necrosis Muscular Idiopática) y ha sido reportado en diversas especies de crustáceos peneidos (Johnson 1995), y M. rosenbergii (Tonguthai 1997, Venugopal et al. 2003) y está asociada a niveles extremos de estrés debido a condiciones ambientales adversas; e) Los juveniles que aparentemente se mantienen por periodos prolongados (días) detenidos en su migración por la barrera, adquieren un aumento de talla y peso, lo que los hace menos capaces de llevar a cabo el escalamiento de las paredes.

Los efectos negativos registrados y observados en la barrera de estudio, son ocasionados por dimensiones físicas de apenas $1,03 \mathrm{~m}$ de altura, con 1,50 m de largo y 1,30 $\mathrm{m}$ de ancho. De acuerdo con estas dimensiones se puede considerar a esta barrera como una presa pequeña pues no rebasa los $15 \mathrm{~m}$ de altura. La Comisión Internacional de Presas Grandes (ICOLD 1998) clasifica a las presas grandes como aquellas con una altura mayor a $15 \mathrm{~m}$. Estimaciones sugieren que existen 37641 presas grandes en el mundo (ICOLD 2013), aquellas que no cuentan con aliviaderos (vertederos), escalas o rampas para el pase de la fauna migratoria, presentan una completa ausencia de camarones anfídromos en los embalses (parte alta), especies que si se encontraban antes de su construcción (Holmquist et al. 1998). Sin embargo, en el mundo existen aproximadamente 800000 presas pequeñas que tienen de tres a cuatro veces el área de embalse de las presas grandes (Rosenberg et al. 2000). Bauer (2011b) y March et al. (2003) consideran a las presas grandes y pequeñas como la alteración humana más dramática y significativa causante del bloqueo de las rutas migratorias en los hábitats de los camarones anfídromos. A pesar de esto, en el resto del mundo, la poca atención científica se ha centrado en los efectos de las presas grandes dejando a un lado las presas pequeñas (March et al. 2003).
Las consecuencias de presas grandes y pequeñas, o de cualquier otro tipo de barrera antrópica sobre la fauna anfídroma pueden ser de gran alcance, no únicamente en las abundancias relativas de las especies migrando en ríos o arroyos ni en la distribución de peces o langostinos, por ejemplo, en muchas islas tropicales, los langostinos anfídromos representan la mayoría de la producción secundaria (Bright 1982) y afectan significativamente varios aspectos de las comunidades bentónicas y procesos de los ecosistemas (Pringle et al. 1999). Exclusiones experimentales a pequeña escala de langostinos resultaron en un aumento de la cantidad y calidad de la materia orgánica (Pringle et al. 1999), aumento de la cubierta de sedimentos, cambios en la composición de ensamblajes y biomasas de insectos y algas (March et al. 2002), y en el decremento en las tasas de procesamiento de basura (March et al. 2001). Con este estudio se evidencia el hecho de que pequeñas barreras antrópicas ocasionan altos impactos negativos sobre la migración río arriba de M. tenellum. Poco se ha estudiado el efecto de pequeñas barreras antrópicas sobre otras especies anfídromas en el planeta, menos aún se conocen las consecuencias ecológicas de la instalación de las barreras grandes (e.g. presas grandes). En México no existen estudios publicados que evalúen el impacto de barreras antrópicas sobre las migraciones de estas especies anfídromas, por lo tanto los efectos que sobre su ecología ocasionan estas modificaciones son desconocidos pero pueden suponerse devastadores.

\section{BIBLIOGRAFÍA}

Bauer, R. T. \& J. Delahoussaye. 2008. Life history migrations of the amphidromous river shrimp Macrobrachium ohione from a continental large river system. Journal of Crustacean Biology 28(4): 622-632.

BAUER, R.T. 2011a. Amphidromy and migrations of freshwater shrimps. I. Costs, benefits, evolutionary origins, and an unusual case of amphidromy. New frontiers in Crustacean Biology: 145-156.

BAUER, R.T. 2011b. Amphidromy and migrations of freshwater shrimps. II. Delivery of hatching larvae to the sea, return juvenile upstream migration, and human impacts. New frontiers in crustacean biology: 157-168.

Benstead, J. P., J. G. March, C. M. Pringle \& F. N. Scatena. 1999. Effects of a low-head dam and water abstraction on migratory tropical stream biota. Ecological Applications 9: 656-668.

BoschI, E.E. 1974. Biología de los crustáceos cultivables en América Latina. Simposio FAO/CARPAS/6/74/SR-7 sobre Acuicultura en América Latina, Montevideo (Uruguay), 26 Nov 1974. Bibliography 18-22.

BRIGHT, G.R. 1982. Secondary benthic production in a tropical island stream. Limnology and Oceanography 27: 472-480.

Dobrick, L.A. 1958. Screening method for glucose of blood serum utilizing glucose oxidase and an indophenol indicator. Journal Biological Chemistry 231: 403-409. 
EspinosA, J.L. 1986. El Langostino: Un alimento en peligro. Serie medio ambiente en Coatzacoalcos, Volumen X. Centro de Ecodesarrollo. D.F., México. 96 p.

Espinosa-chaurand, L.D., M. Vargas-ceballos, M. Guzmánarroyo, H. Nolasco-soria, O. Carrillo-Farnés, O. Chong-Carrillo \& F. Vega-Villasante. 2011. Biología y cultivo de Macrobrachium tenellum: Estado del arte. Hidrobiológica 21(2): 99-117.

Guzmán, A.M., J.G. Rojas \& L. G. GonzÁLez. 1982. Ciclo anual de maduración y reproducción del "chacal" Macrobrachium tenellum (Smith 1871) y su relación con factores ambientales en las lagunas costeras de Mitla y Tres Palos, Gro. México. (Decapoda: Palaemonidae). Anales del Instituto de Ciencias del Mar y Limnología, Universidad Autónoma de México 9 (1): 67-80.

GuzmÁn-Arroyo, M. 1987. Biología, ecología y pesca del langostino Macrobrachium tenellum (Smith, 1871), en lagunas costeras del estado de Guerrero, México. Tesis de Doctorado en Ciencias del Mar (Oceanografía Biológica y Pesquera), Instituto de Ciencias del Mar y Limnología, Colegio de Ciencias y Humanidades, UNAM. D.F., México. 319 p.

Hamano, T. \& K. I. Hayashi. 1992. Ecology of an atyid shrimp Caridina japonica (DeMan 1892) migrating to upstream habitats in the Shiwagi Rivulet, Tokushima prefecture. Researches on Crustacea 21: 1-13.

Hartmann, G. 1958. Apuntes sobre la biología de camarón de río, Cryphiops caementarius (Molina) Palaemonidae, Decapoda. Pesca y Casa. 8: 15-28.

Holmquist, J. G., J. M. Schmidt-Gengenbach \& B. BuchananYoshioka. 1998. High dams and marine-freshwater linkages: effects on native and introduced fauna in the Caribbean. Conservation Biology 12: 621-630.

IBRAHIM, K. H. 1962. Observations on the fishery and biology of the freshwater prawn Macrobrachium malcomsonii. Milne Edwards in River Godvari. Indian Journal of Fisheries 9: 433- 467.

(ICOLD) International Commision on Large Dams.1998. World Register of Dams. Paris: ICOLD.

(ICOLD) International Commision on Large Dams.2013.World Register of Dams. Paris: ICOLD.

Jaime-Ceballos, B., J. Gálindo-López, E. Laria-Lamela, F. Cupul-Magaña \& F. Vega-Villasante. 2008. Traslado de postlarvas de Litopenaeus vannamei (Boone, 1931) a diferentes tiempos, salinidades y densidades y su efecto en la supervivencia y algunos marcadores bioquímicos.
Revista de Biología Marina y Oceanografía 43(3): 681686.

JAYACHANDRAN, K.V. 2001. Palaemonid prawns: Biodiversity, Taxonomy, Biology and Management. Science Publishers, Inc., Reino Unido. 624 p.

Johnson, S.K. 1995. Handbook of shrimp diseases. Department of wildlife and fisheries sciences. Texas A\&M University.

Kikkert, D. A., T. A. Crowl \& A. P. Covich. 2009. Upstream migration of amphidromous shrimp in the Luquillo Experimental Forest, Puerto Rico: temporal patterns and environmental cues. Journal of the North American Benthological Society 28(1): 233-246.

March, J.G., J.P. Benstead, C.M. Pringle and M.R. Ruebel. 2001. Linking shrimp assemblages with rates of detrital processing along an elevational gradient in a tropical stream. Canadian Journal of Fisheries and Aquatic Sciences 58: 470-478.

March, J.G., C.M. Pringle, M.J. Townsend and A.I. Wilson. 2002. Effects of freshwater shrimp assemblages on benthic communities along an altitudinal gradient of a tropical island stream. Freshwater Biology 47: 377-390.

March, J. G., J. P. Benstead, C.M. Pringle \& F. N. Scatena. 2003. Damming tropical island streams: problems, solutions, alternatives. Bioscience 53: 1069-1078.

McDowall, R. M. 1992. Diadromy: origins and definitions of terminology. Copeia 1992: 248-251.

Pringle, C.M., N. Hemphill, W.H. McDowell, A. Bednarek AND J.G. March. 1999. Linking species and ecosystems: Effects of different macrobiotic assemblages on interstream differences in benthic organic matter. Ecology 80: $1860-1872$.

ROMÁN-CONTRERAS, R. 1979. Contribución al conocimiento de la biología y ecología de Macrobrachium tenellum (Smith 1871) (Crustacea, Decapoda, Palaemonidae). Anales del Centro de Ciencias del Mar y Limnología 6(2): 137-160.

Rosenberg, D.M., P. Mccully \& C.M. Pringle. 2000. Globalscale environmental effects of hydrological alterations. BioScience 50: 746-751.

Tonguthai, K. 1997. Diseases of the freshwater prawn, Macrobrachium rosenbergii. AAHRI Newsletter Article 4 (2): 1-4.

Venugopal, G., A. Mandal, N.K. Chadha \& M. Ali. 2003. Growth and survival of Macrobrachium rosenbergii with reference to temperature and hardness. Indian Journal of Fisheries 50(3): 373-378.

Recibido: 02.04.13

Aceptado: 03.09.13 